AIP Applied Physics

\title{
Investigation of the recombination mechanism of excess carriers in undoped BaSi2 films on silicon
}

K. O. Hara, N. Usami, K. Toh, M. Baba, K. Toko et al.

Citation: J. Appl. Phys. 112, 083108 (2012); doi: 10.1063/1.4759246

View online: http://dx.doi.org/10.1063/1.4759246

View Table of Contents: http://jap.aip.org/resource/1/JAPIAU/v112/i8

Published by the American Institute of Physics.

\section{Related Articles}

Growth of controllable $\mathrm{ZnO}$ film by atomic layer deposition technique via inductively coupled plasma treatment J. Appl. Phys. 112, 124102 (2012)

Voltage stress induced reversible diode behavior in pentacene thin films

J. Chem. Phys. 137, 234703 (2012)

Large-area GaN n-core/p-shell arrays fabricated using top-down etching and selective epitaxial overgrowth Appl. Phys. Lett. 101, 241119 (2012)

Growth of pseudomorphic structures through organic epitaxy

J. Chem. Phys. 137, 224703 (2012)

Site preference of cation vacancies in $\mathrm{Mn}$-doped $\mathrm{Ga} 2 \mathrm{O} 3$ with defective spinel structure

Appl. Phys. Lett. 101, 241906 (2012)

\section{Additional information on J. Appl. Phys.}

Journal Homepage: http://jap.aip.org/

Journal Information: http://jap.aip.org/about/about_the_journal

Top downloads: http://jap.aip.org/features/most_downloaded

Information for Authors: http://jap.aip.org/authors

\section{ADVERTISEMENT}

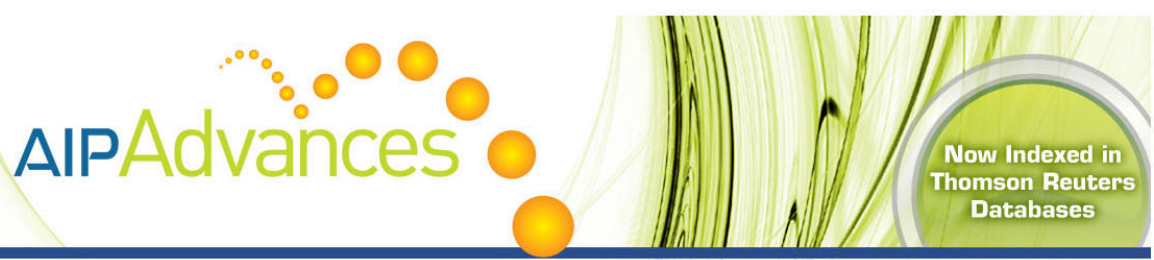

\section{Explore AIP's open access journal: Rapid publication \\ - Article-level metrics \\ Post-publication rating and commenting}




\title{
Investigation of the recombination mechanism of excess carriers in undoped $\mathrm{BaSi}_{2}$ films on silicon
}

\author{
K. O. Hara, ${ }^{1,2, a)}$ N. Usami, ${ }^{1,2}$ K. Toh, ${ }^{3}$ M. Baba, ${ }^{3}$ K. Toko, ${ }^{3}$ and T. Suemasu ${ }^{2,3}$ \\ ${ }^{1}$ Institute for Materials Research, Tohoku University, Sendai 980-8577, Japan \\ ${ }^{2}$ Core Research for Evolutional Science and Technology, Japan Science and Technology Agency, Chiyoda, \\ Tokyo 102-0075, Japan \\ ${ }^{3}$ Institute of Applied Physics, University of Tsukuba, Tsukuba, Ibaraki 305-8573, Japan
}

(Received 17 May 2012; accepted 25 September 2012; published online 23 October 2012)

\begin{abstract}
Excess-carrier recombination mechanisms in undoped $\mathrm{BaSi}_{2}$ epitaxial films grown by molecular beam epitaxy on n-type silicon substrates have been studied by the microwave-detected photoconductivity decay measurement. The measured excess-carrier decay is multiexponential, and we divided it into three parts in terms of the decay rate. Measurement with various excitation laser intensities indicates that initial rapid decay is due to Auger recombination, while the second decay mode with approximately constant decay to Shockley-Read-Hall recombination. Slow decay of the third decay mode is attributed to the carrier trapping effect. To analyze Shockley-Read-Hall recombination, the formulae are developed to calculate the effective lifetime (time constant of decay) from average carrier concentration. The measurement on the films with the thickness of $50-600 \mathrm{~nm}$ shows that the decay due to Shockley-Read-Hall recombination is the slower in the thicker films, which is consistent with the formulae. By fitting the calculated effective lifetime to experimental ones, the recombination probability is extracted. The recombination probability is found to be positively correlated with the full width at half-maximum of the X-ray rocking curves, suggesting that dislocations are acting as recombination centers. (C) 2012 American Institute of Physics. [http://dx.doi.org/10.1063/1.4759246]
\end{abstract}

\section{INTRODUCTION}

Orthorhombic barium disilicide $\left(\mathrm{BaSi}_{2}\right)$ is attracting much attention as a novel active-layer material for thin-film solar cells because it has an appropriate energy gap for singlejunction solar cells and a high absorption coefficient. The energy gap is reported to be $1.3 \mathrm{eV},{ }^{1,2}$ and it can be adjusted to the ideal level $(1.4 \mathrm{eV})$ by alloying with $\mathrm{Sr}^{3}$ The absorption coefficient is $3 \times 10^{4}-1 \times 10^{5} \mathrm{~cm}^{-1}$ at $1.5 \mathrm{eV},{ }^{1,2}$ which is several ten times higher than that of crystalline silicon. The abundance of the constituent atoms, $\mathrm{Ba}$ and $\mathrm{Si}$, in earth's crust is also attractive from the viewpoint of large-scale production. Photoresponsivity corresponding to the internal quantum efficiency of more than $70 \%$ has actually been observed. ${ }^{4}$ In addition, $\mathrm{n}$ - and p-type doping of $\mathrm{BaSi}_{2}$, which is the prerequisite to realize a $\mathrm{BaSi}_{2}$-based solar cell, has also been realized. ${ }^{5-7}$

Excess carrier lifetime is an important physical property and influences the performance of most of electronic devices. In particular, the quality of silicon for solar cells is often evaluated by the minority-carrier lifetime. Thus, the carrier lifetime in $\mathrm{BaSi}_{2}$ should be revealed to consider the potential of $\mathrm{BaSi}_{2}$ as an active-layer material for solar cells. In addition, the knowledge of excess-carrier recombination processes can be extracted from the carrier lifetime, which will contribute as a fundamental knowledge to improve the material quality of $\mathrm{BaSi}_{2}$. The microwave-detected photoconductivity decay $(\mu-\mathrm{PCD})$ method is widely used to determine the carrier lifetime in silicon. The aim of this paper is

\footnotetext{
a) Author to whom correspondence should be addressed. Electronic mail: k-hara@imr.tohoku.ac.jp.
}

to investigate the excess carrier lifetime by the $\mu$-PCD method and to elucidate the carrier recombination mechanisms in $\mathrm{BaSi}_{2}$.

We used the undoped $\mathrm{BaSi}_{2}$ films to reveal highest lifetimes of $\mathrm{BaSi}_{2}$ epitaxial thin films. The undoped $\mathrm{BaSi}_{2}$ epitaxial film is n-type with the electron concentration of $5 \times 10^{15} \mathrm{~cm}^{-3}{ }^{1}$. Since the majority-carrier concentration is low and the absorption coefficient is high, it is difficult to satisfy low-injection conditions in thin films and to determine minority-carrier lifetime. Moreover, due to the large difference in work function between the $\mathrm{BaSi}_{2}$ layer and silicon substrate, an internal electric field is formed in the $\mathrm{BaSi}_{2}$ layer. This electric field makes photoexcited electrons and holes separated. Thus, the model considering high injection and carrier separation is developed and the photoconductivity decay data are analyzed.

\section{EXPERIMENTAL METHODS}

The $a$-axis-oriented $\mathrm{BaSi}_{2}$ epitaxial films were prepared on $\operatorname{Si}(111)$ substrates (n-type, $1000 \Omega \cdot \mathrm{cm}$ in resistivity) by reactive deposition epitaxy (RDE; Ba deposition on hot $\mathrm{Si}$ ) followed by molecular beam epitaxy (MBE; co-deposition of $\mathrm{Ba}$ and $\mathrm{Si}$ ). An ion-pumped MBE system equipped with a standard Knudsen cell for Ba and an electron-beam evaporation source for $\mathrm{Si}$ was used. The RDE process was carried out for the deposition of a $\mathrm{BaSi}_{2}$ template layer prior to the subsequent MBE process. The details of the growth procedure are described elsewhere. ${ }^{8}$

Excess carrier lifetime was investigated by the $\mu$-PCD method using a semiconductor wafer lifetime measuring 
system (KOBELCO, LTA-1512EP). Carriers were generated by a $5 \mathrm{~ns}$ laser pulse with a wavelength of $349 \mathrm{~nm}$. More than $95 \%$ of the light is absorbed within the $\mathrm{BaSi}_{2}$ layer. The spot size of the laser is $2 \mathrm{~mm}$ in diameter. To investigate the effect of carrier injection level on carrier lifetime, laser intensity was varied in the range $1.1 \times 10^{2}-1.3 \times 10^{5} \mathrm{~W} / \mathrm{cm}^{2}$, corresponding to the area photon density in the range $9.8 \times 10^{11}-1.1 \times 10^{15} \mathrm{~cm}^{-2}$, assuming no reflection at the $\mathrm{BaSi}_{2}$ surface. Photoconductivity decay was monitored by the reflectivity of microwave with the frequency of $26 \mathrm{GHz}$. High-sensitivity measurement was realized by the differential detection of the reflected microwave intensity between the areas with and without laser irradiation.

Since the difference in electron affinity is large between $\mathrm{BaSi}_{2}(3.2 \mathrm{eV})$ and silicon $(4.0 \mathrm{eV}){ }^{9}$ band bending is supposed to occur around the $\mathrm{BaSi}_{2} / \mathrm{Si}$ interface. To consider the effects of band bending on carrier lifetime, the band diagrams of the sample structures studied were calculated with the wxAMPS software, ${ }^{10}$ which is a solar cell simulation tool. In the calculation, the effective mass of electrons and the dielectric constant of $\mathrm{BaSi}_{2}$ were assumed to be the same as those of silicon. ${ }^{11,12}$ The band diagrams were used to analyze the photoconductivity decay curves.

To compare with carrier lifetime, the crystalline quality of the $\mathrm{BaSi}_{2}$ layer was investigated by the X-ray rockingcurve measurement using a Bruker Discover D8 diffractometer with a four-bounce $\mathrm{Ge}(220)$ monochromator. The 600 diffraction line of $\mathrm{BaSi}_{2}$ was used.

\section{RESULTS AND DISCUSSION}

\section{A. Effects of laser intensity on photoconductivity decay}

The calculated band diagram of the undoped 130-nmthick $\mathrm{BaSi}_{2}$ film on n-type silicon is shown in Fig. 1. It is seen that there is a constant electric field in the whole $\mathrm{BaSi}_{2}$ layer. The photoexcited carriers are, therefore, expected to be separated in the $\mathrm{BaSi}_{2}$ film. It should also be noted that the conduction type of $\mathrm{BaSi}_{2}$ around the interface is p-type due to the migration of a large number of electrons into the

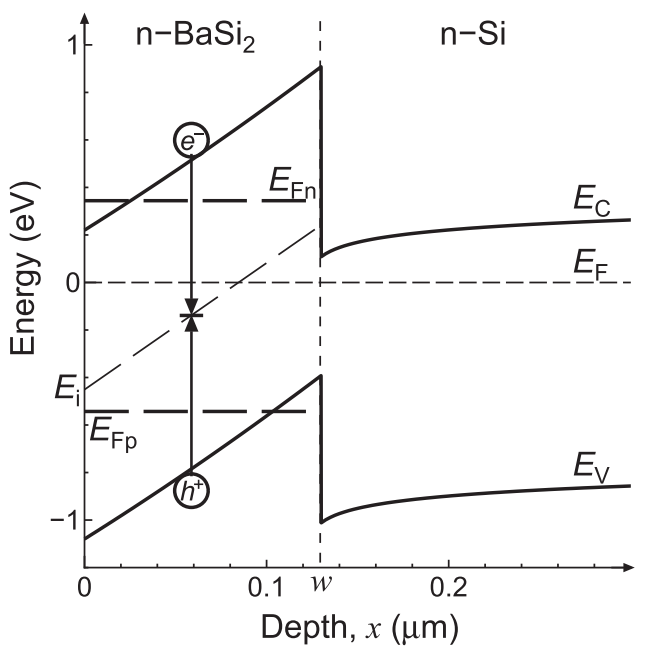

FIG. 1. Band diagram of the 130-nm-thick $\mathrm{BaSi}_{2}$ film on n-type silicon calculated by the wxAMPS software. ${ }^{10}$ The schematic figure of SRH recombination with schematic quasi-Fermi levels, $E_{\mathrm{Fn}}$ and $E_{\mathrm{Fp}}$, is also shown.
Si substrate. This fact that the $\mathrm{BaSi}_{2}$ layer consists of the pn junction of $\mathrm{BaSi}_{2}$ will be used in the later analyses. In the calculation, we have assumed that the effective mass of electrons and the dielectric constant of $\mathrm{BaSi}_{2}$ are the same as those of silicon. This assumption affects the magnitude of the internal electric field.

Figure 2 shows the photoconductivity decay curves of the 130-nm-thick $\mathrm{BaSi}_{2}$ film with excitation laser intensities of $1.1 \times 10^{2}-1.3 \times 10^{5} \mathrm{~W} / \mathrm{cm}^{2}$. In all curves, decay rate changes with time. With high laser intensities [Figs. 2(a) and 2(b)], decay can be divided into three modes in terms of decay rate: Initial rapid decay $(t<10 \mu \mathrm{s})$, approximately constant decay $[t<45$ (a) and $35 \mu$ s (b)], and later slow decay. In contrast, approximately constant decay is not observed with low laser intensities [Figs. 2(c) and 2(d)]. Possible dominant recombination modes in $\mathrm{BaSi}_{2}$ with indirect band gap $^{13,14}$ are Shockley-Read-Hall (SRH) and Auger recombinations while radiative recombination is less probable than the others. In the following paragraphs, the recombination mechanism corresponding to each decay mode will be discussed on the basis of the laser intensity dependence of the decay curves.

In the early stage where carrier concentration is high, $\mathrm{Au}-$ ger recombination can be a dominant process. Assuming that the excitation light is fully absorbed in $\mathrm{BaSi}_{2}$ without reflection, the initial excess-carrier concentration at the surface is in the range $8.9 \times 10^{17}-1.0 \times 10^{21} \mathrm{~cm}^{-3}$, which is more than two orders of magnitude higher than majority-carrier concentration at equilibrium in undoped $\mathrm{BaSi}_{2}\left(5 \times 10^{15} \mathrm{~cm}^{-3}\right)$. Another possible origin of the initial rapid decay is surface SRH recombination. To investigate the origin, the early-stage photoconductivity decay was measured with a small time step, which is shown in Fig. 3. It is clearly observed that initial decay rate becomes slow with decreasing the laser intensity from $1.3 \times 10^{5} \mathrm{~W} / \mathrm{cm}^{2}$ [Fig. 3(a)] to $1.1 \times 10^{2} \mathrm{~W} / \mathrm{cm}^{2}$ [Fig. 3(d)]. This dependence of decay rate on the carrierinjection level shows that the initial rapid decay is mainly originated from Auger recombination.

Since there is an internal electric field in $\mathrm{BaSi}_{2}$ as shown in Fig. 1, carrier separation probably occurs at the same time

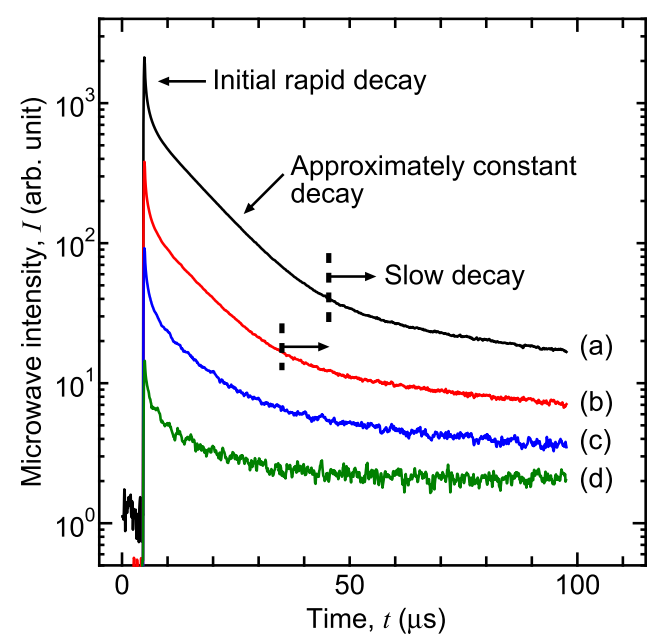

FIG. 2. Photoconductivity decay curves of the 130 -nm-thick $\mathrm{BaSi}_{2}$ film with the laser intensities of (a) $1.3 \times 10^{5}$, (b) $1.3 \times 10^{4}$, (c) $1.1 \times 10^{3}$, and (d) $1.1 \times 10^{2} \mathrm{~W} / \mathrm{cm}^{2}$. 


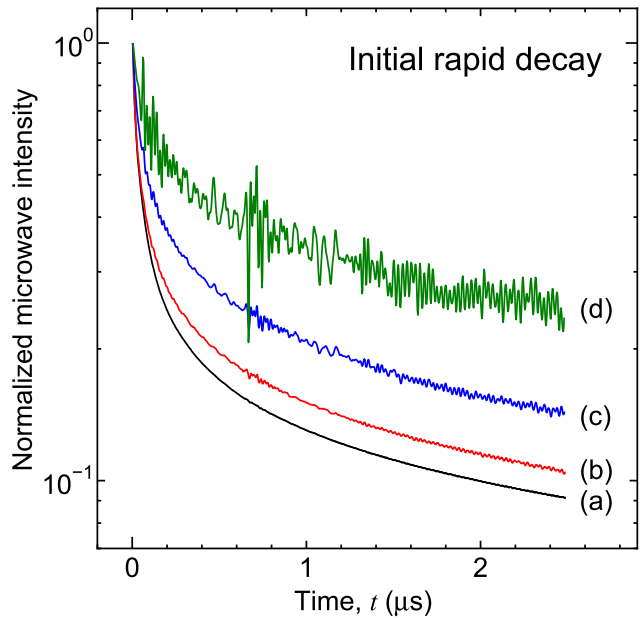

FIG. 3. Early-stage $\mu$-PCD curves of the $130-$ nm-thick $\mathrm{BaSi}_{2}$ film with laser intensities of (a) $1.3 \times 10^{5}$, (b) $1.3 \times 10^{4}$, (c) $1.1 \times 10^{3}$, and (d) 1.1 $\times 10^{2} \mathrm{~W} / \mathrm{cm}^{2}$. Microwave intensities were normalized by the peak values.

as Auger recombination. As a result, electrons and holes become in high concentration around the surface and interface, respectively. After carrier separation, Auger recombination would become slow, and next possible recombination mode is SRH recombination. As schematically shown in Fig. 1 together with the band diagram, SRH recombination would occur mainly at the middle of the film, where both types of carriers are in similar concentration.

It is seen in Fig. 2 that there are two decay modes for SRH recombination with high laser intensities: approximately constant and slow decays [Figs. 2(a) and 2(b)]. Similar slowdown of decay has been reported for silicon and has been attributed to carrier trapping. ${ }^{15-17}$ That is, the carrier trapping which does not lead to carrier recombination prevents carriers from reaching recombination centers and lengthens carrier lifetime. Slow decay with the carrier trapping effect starts at earlier time with lower laser intensity [Figs. 2(a) and 2(b)]. This is probably because there would not be many free (untrapped) carriers which can reach recombination centers when the amount of excess carriers is small. Thus, with even lower laser intensities [Figs. 2(c) and 2(d)], slow decay starts soon after carrier separation without approximately constant decay.

$\mathrm{SRH}$ recombination at the interface between the holes in $\mathrm{BaSi}_{2}$ and the electrons in silicon is also probable. Total amount of this interface recombination is, however, restricted to the carrier amount in silicon $\left(\sim 4.5 \times 10^{12} \mathrm{~cm}^{-3} \times 3.8 \times 10^{-2} \mathrm{~cm}\right.$ $=1.7 \times 10^{11} \mathrm{~cm}^{-2}$ ) and is smaller than the number of excess carriers in $\mathrm{BaSi}_{2}$ under the present conditions.

\section{B. Numerical analysis of SRH recombination}

The above experimental results show that effective lifetime $\left(\tau_{\text {eff }}\right)$ is a function of time. In such a case, $\tau_{\text {eff }}$ can be obtained by differentiating the excess-carrier concentration with respect to time. ${ }^{18,19}$ By assuming that the reflected microwave intensity $(I)$ of $\mu$-PCD is proportional to the excess-carrier concentration, $\tau_{\text {eff }}$ can be calculated as

$$
\frac{1}{\tau_{\text {eff }}}=-\frac{1}{I} \frac{\mathrm{d} I}{\mathrm{~d} t} .
$$

Though this assumption is usually valid under low-injection conditions, it is a reasonable approximation also in this study of high-injection conditions because the peak values of the decay curves increase almost linearly with the increase in laser intensity.

Figure 4 shows the $\tau_{\text {eff }}$ values calculated for laser intensities of $2.5 \times 10^{4}-1.3 \times 10^{5} \mathrm{~W} / \mathrm{cm}^{2}$ by means of Eq. (1) as a function of $I$. Before differentiation, a moving average of the decay curve was calculated to reduce noise. The $\tau_{\text {eff }}$ value increases with the decrease in $I$ as a general trend. It is seen that each series of $\tau_{\text {eff }}$ can be divided into three parts, which correspond to Auger recombination, and SRH recombinations without and with the carrier trapping effect, according to the decay curves. Of the three carrier-recombination modes, SRH recombination without the carrier trapping effect reflects crystalline quality and usually determines the minority-carrier lifetime, which should be high to achieve high solar-cell efficiency. Thus, this recombination mode is analyzed in detail to extract the carrier-capture probability by recombination centers.

It should be recalled here that the pn junction of $\mathrm{BaSi}_{2}$ is formed in the $\mathrm{BaSi}_{2}$ layer (Fig. 1). The SRH recombination velocity $\left(R_{\mathrm{np}}\right)$ can, therefore, be represented by that of the pn junction as ${ }^{20}$

$$
R_{\mathrm{np}}=\frac{1}{2} C_{\mathrm{np}} n_{\mathrm{i}}\left\{\exp \left(\frac{\Delta E_{\mathrm{F}}}{2 k T}\right)-1\right\},
$$

with the assumption of one deep trap, the energy level of which falls on the intrinsic Fermi level. In Eq. (2), $n_{\mathrm{i}}$ is carrier concentration in an intrinsic specimen, $\Delta E_{\mathrm{F}}$ is the difference of the quasi-Fermi levels of electrons and holes, $k$ is the Boltzmann constant, and $T$ is the temperature. $C_{\text {np }}$ denotes the carrier capture probability by deep traps, which can also be regarded as the recombination probability of electrons

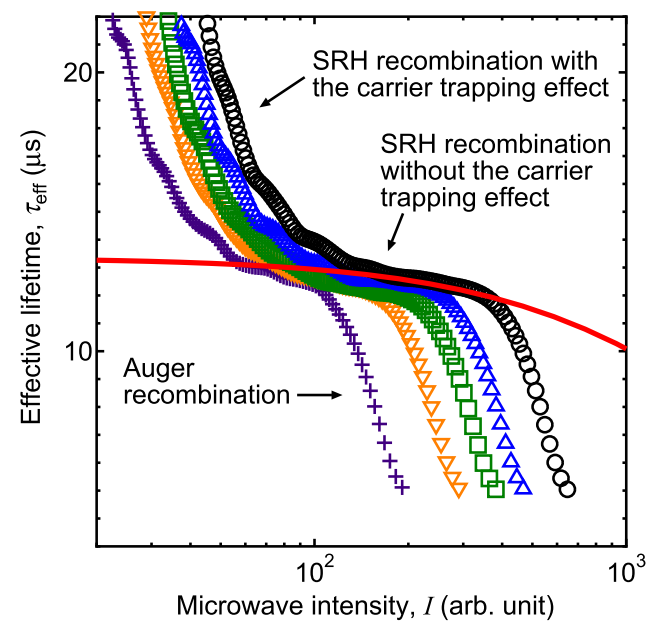

FIG. 4. Effective lifetime ( $\tau_{\text {eff }}$ ) of the 130-nm-thick $\mathrm{BaSi}_{2}$ film as functions of the reflected microwave intensity $(I)$. Five curves with different laser intensities of $2.5 \times 10^{4}-1.3 \times 10^{5} \mathrm{~W} / \mathrm{cm}^{2}$ are displayed: $\bigcirc$ (black) for the laser intensity of $1.3 \times 10^{5} \mathrm{~W} / \mathrm{cm}^{2} ; \triangle$ (blue) for $8.8 \times 10^{4} \mathrm{~W} / \mathrm{cm}^{2} ; \square$ (green) for $6.1 \times 10^{4} \mathrm{~W} / \mathrm{cm}^{2} ; \nabla$ (orange) for $4.3 \times 10^{4} \mathrm{~W} / \mathrm{cm}^{2} ;+$ (purple) for $2.5 \times 10^{4} \mathrm{~W} / \mathrm{cm}^{2}$. Red curve corresponds to the calculated $\tau_{\text {eff }}$ curve fitted to the experimental $\tau_{\text {eff }}$ values of SRH recombination without the carrier trapping effect. 
and holes injected into highly p-type and n-type specimens, respectively. ${ }^{21} \tau_{\text {eff }}$ is obtained from $R_{\mathrm{np}}$ as

$$
\tau_{\mathrm{eff}}=\frac{n_{\mathrm{ave}}}{R_{\mathrm{np}}},
$$

where $n_{\text {ave }}$ is average carrier concentration in the $\mathrm{BaSi}_{2}$ film. Here, average excess-carrier concentration was approximated by average carrier concentration considering highinjection conditions. $n_{\text {ave }}$ is derived from the integrations with respect to energy $(E)$ and position $(x)$ of the product of the density of states $(D)$ and the Fermi-Dirac distribution function $(f)$ as

$$
n_{\mathrm{ave}}=\frac{1}{w} \int_{0}^{w} \int_{E_{\mathrm{C}}}^{\infty} D(E, x) f(E, x) \mathrm{d} E \mathrm{~d} x,
$$

where $w$ and $E_{\mathrm{C}}$ are the film thickness and the conduction band minimum, respectively. As seen in Fig. 1, $E_{\mathrm{C}}$ can be approximated by a linear function of position, $a x+b$. The coefficient of determination (the goodness of fit) of the linear fitting is 1.0000 for the 130 -nm-thick $\mathrm{BaSi}_{2}$ layer. Under this approximation, the integration with respect to $x$ in Eq. (4) can be analytically calculated and Eq. (4) results in

$$
\begin{aligned}
n_{\mathrm{ave}}= & \frac{8 \pi}{3 a w}\left(\frac{2 m^{*}}{h^{2}}\right)^{\frac{3}{2}}\left\{\int_{b}^{\infty} \frac{(E-b)^{\frac{3}{2}}}{1+\exp \left(\frac{E-E_{\mathrm{Fn}}}{k T}\right)} \mathrm{d} E\right. \\
& \left.-\int_{a w+b}^{\infty} \frac{(E-a w-b)^{\frac{3}{2}}}{1+\exp \left(\frac{E-E_{\mathrm{F}}}{k T}\right)} \mathrm{d} E\right\}
\end{aligned}
$$

where $h$ and $E_{\mathrm{Fn}}$ are the Planck constant and the quasi-Fermi level of electrons, respectively. The effective mass of electrons $\left(m^{*}\right)$ was assumed to be equal to that in silicon. ${ }^{11}$ It was also assumed that the conduction and valence band levels did not change by carrier separation. Now, by numerically solving Eq. (5), $E_{\mathrm{Fn}}$ can be determined for a given $n_{\text {ave }}$ value. And, $R_{\mathrm{np}}$ can be calculated by using Eq. (2) from $E_{\mathrm{Fn}}$ and the $C_{\mathrm{np}}$ parameter. In consequence, for a given $C_{\mathrm{np}}$ value, $\tau_{\text {eff }}$ can be calculated using Eqs. (2), (3), and (5) as a function of $n_{\text {ave }}$.

In Fig. $4, \tau_{\text {eff }}$ of SRH recombination without the carrier trapping effect is seen to be determined by $I$, and hence, $n_{\text {ave }}$, regardless of the laser intensity. This is consistent with the formulae developed above. To compare the calculated and experimental $\tau_{\text {eff }}$ values, $I$ was assumed to be proportional to $n_{\text {ave }}$, according to the approximations to calculate $\tau_{\text {eff }}$ in Eqs. (1) and (3). By optimizing the proportionality constant between $I$ and $n_{\text {ave }}$, and $C_{\mathrm{np}}$, the $\tau_{\mathrm{eff}}$ curve was fitted to the experimental $\tau_{\text {eff }}$ values of the SRH recombination without the carrier trapping effect in Fig. 4. The optimized $C_{\mathrm{np}}$ value is $3 \mathrm{~ns}^{-1}$, which corresponds to the carrier lifetime of $0.3 \mathrm{~ns}$. This is the carrier lifetime of electrons or holes in a highly $\mathrm{p}$ - or n-type doped specimen, and minority-carrier lifetime is expected to be higher than this value.

Since we have made several assumptions, the obtained $C_{\mathrm{np}}$ value contains uncertainty. The effective mass of electrons and dielectric constant of $\mathrm{BaSi}_{2}$ were assumed to be the same as silicon in the band diagram calculation, which affect the magnitude of electric field in the $\mathrm{BaSi}_{2}$ layer. In the derivation of formulae, $E_{\mathrm{C}}$ was assumed that it is not influenced by the spatial separation of excess carriers. The error in $C_{\text {np }}$ accordingly comes mainly from the uncertainty in $a$. We thus calculated $C_{\mathrm{np}}$ with changing $a$ to assess the importance of uncertainty in $a$. As a result, $C_{\mathrm{np}}$ was found to change by 3.3-3.5 times when $a$ changes by $10 \%$, for example. The error in $C_{\mathrm{np}}$ is therefore expected to be large, and thus, we focus only the relative difference of $C_{\mathrm{np}}$ in later analyses.

\section{Effects of $\mathrm{BaSi}_{2}$ film thickness on photoconductivity decay}

Effects of the $\mathrm{BaSi}_{2}$ film thickness (w) on carrier lifetime were investigated for $w=50-600 \mathrm{~nm}$. Figure 5 shows the photoconductivity decay curves with the laser intensity of $1.3 \times 10^{5} \mathrm{~W} / \mathrm{cm}^{2}$. All films except 600 -nm-thick one show three decay modes similarly to the 130-nm-thick film in Fig. 2. In the 600-nm-thick film, constant decay is not observed and the carrier trapping effect plays an important role soon after Auger recombination. This is possibly because the average excess-carrier concentration is low in the thick film and not all of the traps are filled by excess carriers.

It is clearly seen in Fig. 5 that thicker films exhibit slower photoconductivity decay in SRH recombination without the carrier trapping effect. This is because of the following two factors. One is the increase of $E_{\mathrm{Fn}}$ with decreasing $w$. For a given number of excess carriers, $n_{\text {ave }}$ is the higher in the thinner films, which corresponds to the higher $E_{\mathrm{Fn}}$ level. Then, $R_{\mathrm{np}}$ is the larger according to Eq. (2), and thus, $\tau_{\mathrm{eff}}$ is the smaller for the thinner films. The other factor influencing the decay rate of SRH recombination is the difference in the amount of lattice defects acting as recombination centers.

To evaluate the relative amount of recombination centers, the analysis of $\tau_{\text {eff }}$ was performed on the 50 -, 70 -, and 100 -nm-thick films. Figure 6 shows $\tau_{\text {eff }}$ of these films as a function of $I$. All films show the increasing trend of $\tau_{\text {eff }}$ with the decrease of $I$. The $\tau_{\text {eff }}$ curves of 70 - and 100-nm-thick films are seen to oscillate for $\tau_{\text {eff }}>8 \mu \mathrm{s}$. This is because of noise, which is distinct when $I$ is small. By fitting the $\tau_{\text {eff }}$ values calculated by Eqs. (2), (3), and (5) to the experimental

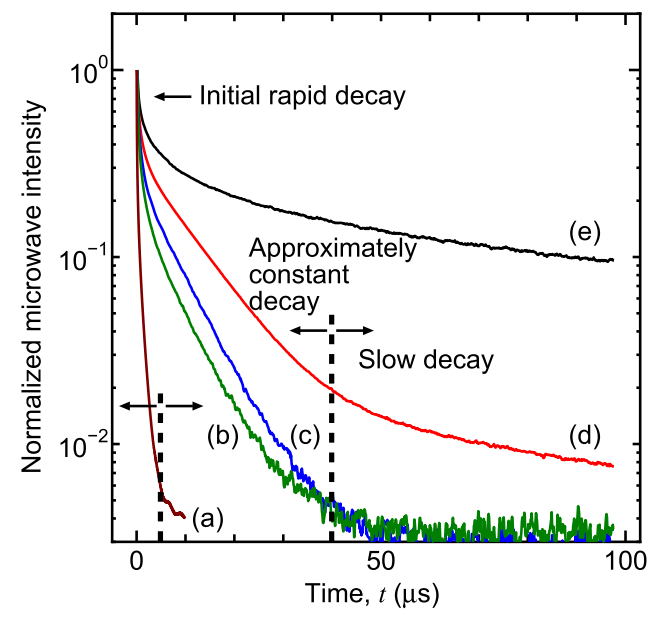

FIG. 5. $\mu$-PCD curves of the (a) 50 -, (b) 70 -, (c) 100 -, (d) 130 -, and (e) 600 -nm-thick $\mathrm{BaSi}_{2}$ films with the laser intensity of $1.3 \times 10^{5} \mathrm{~W} / \mathrm{cm}^{2}$. Microwave intensities were normalized by the peak values. 

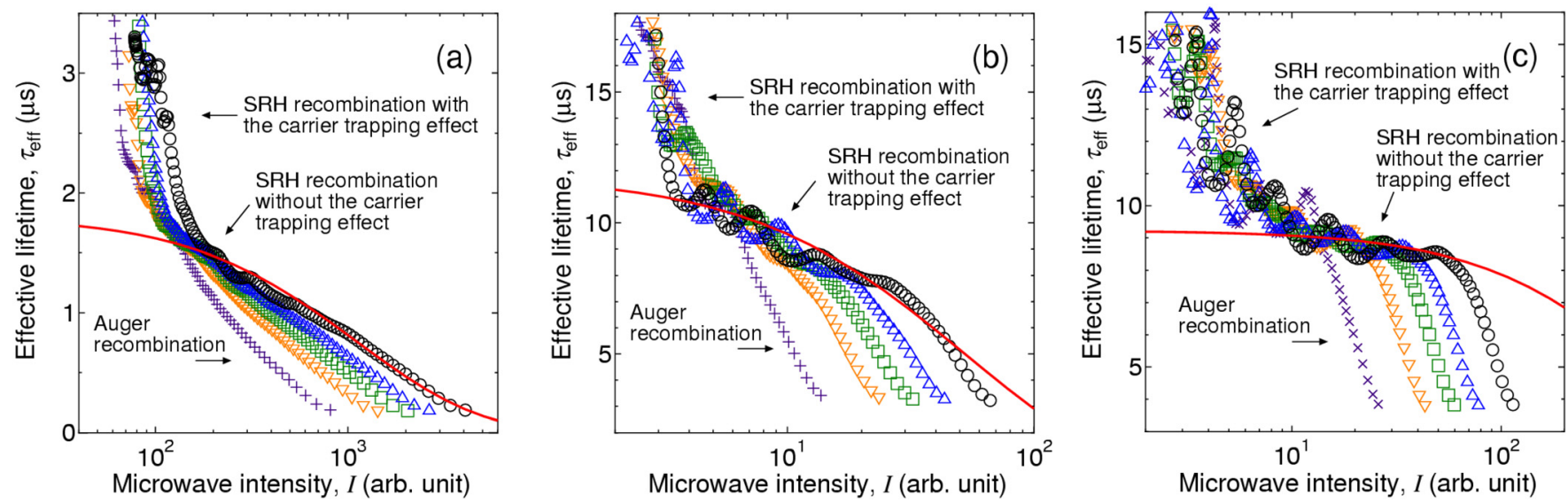

FIG. 6. Effective lifetime ( $\tau_{\text {eff }}$ ) of the (a) 50 -, (b) 70 -, and (c) 100-nm-thick $\mathrm{BaSi}_{2}$ films as functions of the reflected microwave intensity (I). Five curves with different laser intensities of $2.5 \times 10^{4}-1.3 \times 10^{5} \mathrm{~W} / \mathrm{cm}^{2}$ are displayed for each film: $\bigcirc$ (black) for the laser intensity of $1.3 \times 10^{5} \mathrm{~W} / \mathrm{cm}^{2} ; \triangle(\mathrm{blue}$ ) for $8.8 \times 10^{4} \mathrm{~W} / \mathrm{cm}^{2} ; \square$ (green) for $6.1 \times 10^{4} \mathrm{~W} / \mathrm{cm}^{2} ; \nabla$ (orange) for $4.3 \times 10^{4} \mathrm{~W} / \mathrm{cm}^{2} ;+$ (purple) for $2.5 \times 10^{4} \mathrm{~W} / \mathrm{cm}^{2}$. Red curves correspond to the calculated $\tau_{\text {eff }}$ curves fitted to the experimental $\tau_{\text {eff }}$ values of SRH recombination without the carrier trapping effect.

values, $C_{\mathrm{np}}$ and the proportionality constant between $I$ and $n_{\text {ave }}$ are determined. The $a$ and $b$ coefficients used in Eq. (5) were obtained by linear fitting with the coefficients of determination not less than 0.9997 . The determined $C_{\mathrm{np}}$ values are plotted as a function of $w$ in Fig. 7. Error bars indicate the standard deviations calculated from the analysis results for nine different points on the film surface. It is seen that $C_{\mathrm{np}}$ of the 50-nm-thick film is the largest, while the $C_{\mathrm{np}}$ values of the $70-, 100$-, and 130-nm-thick films are similar to each other. This result suggests that the 50-nm-thick film contains larger number of the lattice defects which act as carrier recombination centers.

To investigate the origin of the difference in the $C_{\mathrm{np}}$ values, the X-ray rocking curves were measured for the 50 -, 70 -, 100 -, and 130-nm-thick films. Figure 8 shows the full width at half-maximum (FWHM) of the rocking curves as a function of $w$. The FWHM value of the 50-nm-thick film is larger than the others, indicating that the crystal orientation fluctuation is larger in the 50-nm-thick film than the others. Mosaic structure usually involves dislocations at the boundary, which is the origin of the orientation fluctuation. Thus,

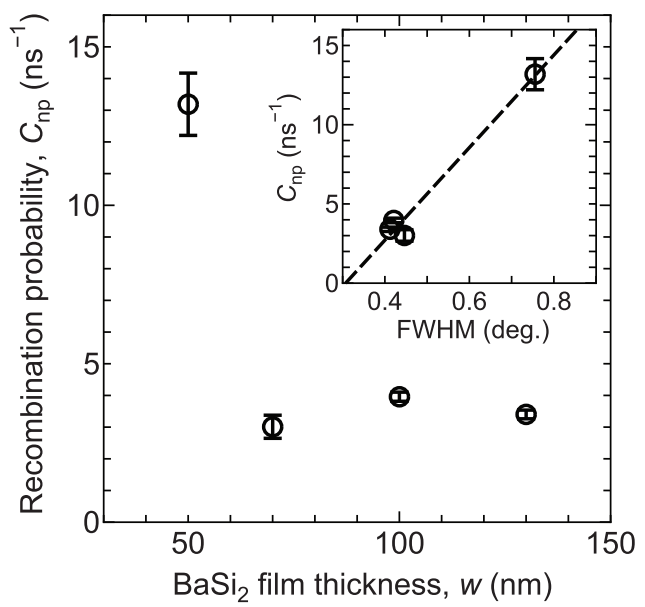

FIG. 7. Recombination probability $\left(C_{\mathrm{np}}\right)$ as a function of the $\mathrm{BaSi}_{2}$ film thickness $(w)$. The inset shows $C_{\mathrm{np}}$ as a function of the FWHM of X-ray rocking curves. The dashed line is the least-square fit to the data. the 50-nm-thick film has higher density of dislocations. Interestingly, the dependency of the FWHM values on $w$ agrees well with $C_{\mathrm{np}}$ in Fig. 7. The inset of Fig. 7 shows $C_{\mathrm{np}}$ as a function of the FWHM of rocking curves. A positive correlation between $C_{\mathrm{np}}$ and FWHM of rocking curves is observed, which suggests that the dislocations act as recombination centers.

Although the uncertainty in $a$ causes a significant error in $C_{\mathrm{np}}$, the analysis result that $C_{\mathrm{np}}$ of the 50 -nm-thick film is larger than the other films is not influenced. We assessed the influence of the uncertainty in $a$ by calculating $C_{\mathrm{np}}$ with intentionally changed $a$ values (up to $50 \%$ increase and decrease). As a result, we obtained similar results that $C_{\mathrm{np}}$ of the 50 -nm-thick film is significantly larger than the others, though the absolute values change by up to several hundred times. Therefore, the conclusion that $C_{\mathrm{np}}$ is largest in the 50-nm-thick film and is correlated to the dislocation density is not affected by the assumptions used. Developed formulae, thus, enable us to evaluate the relative difference of $C_{\mathrm{np}}$ between the $\mathrm{BaSi}_{2}$ thin films with different thickness.

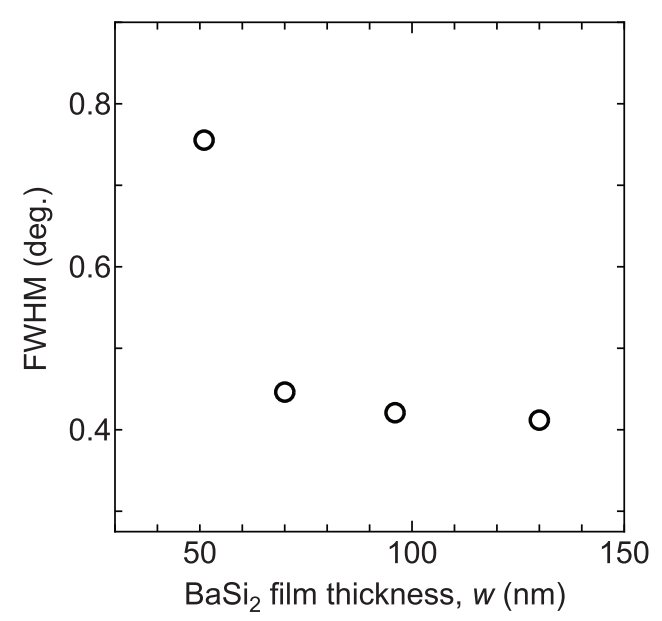

FIG. 8. Full width at half-maximum (FWHM) of the rocking curve of the $\mathrm{BaSi}_{2} 600$ diffraction as a function of the $\mathrm{BaSi}_{2}$ film thickness $(w)$. 


\section{SUMMARY}

We have investigated the excess carrier lifetime in undoped $\mathrm{BaSi}_{2}$ epitaxial films grown by molecular beam epitaxy on high-resistivity n-type silicon by the microwavedetected photoconductivity decay method under high-injection conditions. The photoconductivity decay in undoped $\mathrm{BaSi}_{2}$ epitaxial film was divided into three parts from the viewpoint of the decay rate. Initial rapid decay was attributed to Auger recombination. SRH recombination at the middle of the film prevails in the second part after Auger recombination. Decay becomes slower due to the carrier trapping effect, which corresponds to the third decay mode. To analyze SRH recombination, the formulae were developed to calculate the effective lifetime from average carrier concentration in $\mathrm{BaSi}_{2}$. The experimental result that the thicker films show the longer lifetimes is consistent with the formulae. By comparing the calculated effective lifetime with experimental results, the recombination probability was extracted. According to the $\mathrm{X}$-ray rocking curve measurement, the dislocation density was shown to be correlated with the recombination probability, which suggests that dislocations act as recombination centers.

\section{ACKNOWLEDGMENTS}

This work was supported by CREST of the Japan Science and Technology Agency.
${ }^{1}$ K. Morita, Y. Inomata, and T. Suemasu, Thin Solid Films 508, 363 (2006).

${ }^{2}$ K. Toh, T. Saito, and T. Suemasu, Jpn. J. Appl. Phys., Part 1 50, 068001 (2011).

${ }^{3}$ K. Morita, M. Kobayashi, and T. Suemasu, Jpn. J. Appl. Phys., Part 2 45, L390 (2006).

${ }^{4}$ W. Du, M. Suzuno, M. A. Khan, K. Toh, M. Baba, K. Nakamura, K. Toko, N. Usami, and T. Suemasu, Appl. Phys. Lett. 100, 152114 (2012).

${ }^{5}$ M. Kobayashi, Y. Matsumoto, Y. Ichikawa, D. Tsukada, and T. Suemasu, Appl. Phys. Express 1, 051403 (2008).

${ }^{6}$ M. Ajmal Khan, M. Takeishi, Y. Matsumoto, T. Saito, and T. Suemasu, Phys. Procedia 11, 11 (2011).

${ }^{7}$ M. Takeishi, Y. Matsumoto, R. Sasaki, T. Saito, and T. Suemasu, Phys. Procedia 11, 27 (2011).

${ }^{8}$ Y. Inomata, T. Nakamura, T. Suemasu, and F. Hasegawa, Jpn. J. Appl. Phys., Part 2 43, L478 (2004).

${ }^{9}$ T. Suemasu, K. Morita, M. Kobayashi, M. Saida, and M. Sasaki, Jpn. J. Appl. Phys., Part 2 45, L519 (2006).

${ }^{10}$ Y. Liu, Y. Sun, and A. Rockett, Sol. Energy Mater. Sol. Cells 98, 124 (2012).

${ }^{11}$ H. D. Barber, Solid-State Electron. 10, 1039 (1967).

${ }^{12}$ W. C. Dunlap, Jr. and R. L. Watters, Phys. Rev. 92, 1396 (1953).

${ }^{13}$ T. Nakamura, T. Suemasu, K. Takakura, F. Hasegawa, A. Wakahara, and M. Imai, Appl. Phys. Lett. 81, 1032 (2002).

${ }^{14}$ D. B. Migas, V. L. Shaposhnikov, and V. E. Borisenko, Phys. Status Solidi B 244, 2611 (2007).

${ }^{15}$ J. A. Hornbeck and J. R. Haynes, Phys. Rev. 97, 311 (1955).

${ }^{16}$ D. Macdonald and A. Cuevas, Appl. Phys. Lett. 74, 1710 (1999).

${ }^{17}$ Y. Hu, H. Schøn, Ø. Nielsen, E. Johannes Øvrelid, and L. Arnberg, J. Appl. Phys. 111, 053101 (2012).

${ }^{18}$ E. Yablonovitch and T. Gmitter, Appl. Phys. Lett. 49, 587 (1986).

${ }^{19}$ S. K. Pang and A. Rohatgi, J. Appl. Phys. 74, 5554 (1993).

${ }^{20} \mathrm{P}$. Würfel, Physics of Solar Cells (Wiley Online Library, 2005).

${ }^{21}$ C. T. Sah, R. N. Noyce, and W. Shockley, Proc. IRE 45, 1228 (1957). 